\title{
Exponential bounds on the number of causal triangulations
}

\author{
Bergfinnur Durhuus ${ }^{1}$ \\ Department of Mathematical Sciences \\ University of Copenhagen, Universitetsparken 5 \\ DK-2100 Copenhagen $\varnothing$, Denmark
}

Thordur Jonsson2

Division of Mathematics, The Science Institute

University of Iceland, Dunhaga 3

IS-107 Reykjavik, Iceland

\begin{abstract}
We prove that the number of combinatorially distinct causal 3-dimensional triangulations homeomorphic to the 3-dimensional sphere is bounded by an exponential function of the number of tetrahedra. It is also proven that the number of combinatorially distinct causal 4-dimensional triangulations homeomorphic to the 4-sphere is bounded by an exponential function of the number of 4-simplices provided the number of all combinatorially distinct triangulations of the 3 -sphere is bounded by an exponential function of the number of tetrahedra.
\end{abstract}

\footnotetext{
${ }^{1}$ email: durhuus@math.ku.dk

2email: thjons@hi.is
} 


\section{Introduction}

Random triangulations have been used for over 30 years to construct discrete models of 2-dimensional quantum gravity. For a review of early work in this field, see [1]. In [2] the generalization of these models to three dimensions was considered and it was pointed out that one needs bounds on the number of combinatorially distinct triangulations of a given fixed topology in order for the models to be well defined. More precisely: If $N(V)$ is the number of distinct triangulations of the 3 -sphere, say, by $V$ tetrahedra we need a bound of the form

$$
N(V) \leq C^{V}
$$

for some constant $C$. It is easy to deduce from (1) the corresponding bound for triangulations of the 3-ball or, more generally, the 3-sphere with a number of 3-balls removed.

In [12] the notion of locally constructible triangulations was introduced and such triangulations of $S^{3}$ were shown to obey the bound (1). Subsequently it was proven that not all triangulations of $S^{3}$ are locally constructible [8] and a proof of (1) is still missing. See [10] for a recent result which gives a new sufficient condition for (1) to hold. Monte Carlo simulations of the 3-dimensional gravity models indicate that a bound of the form (1) is valid, see [7, 9].

An alternative approach to discretized quantum gravity in two and higher dimensions is the use of so-called causal triangulations. This is the class of triangulations that we are concerned with in the bulk of this paper. A general definition is given in Section 2. In the case of 3 dimensions they can be described as follows: We first introduce the notion of a causal slice which is a triangulation of $S^{2} \times[0,1]$ with the property that all the vertices lie on the boundary and every tetrahedron has a least one vertex in each of the two boundary components. If $K$ is a causal slice then its boundary $\partial K$ consists of two triangulations of the 2-sphere. We choose to label the boundary components and call one of them the in-boundary and the other one the out-boundary. A causal triangulation $K$ is then defined by a sequence 
$K_{1}, K_{2}, \ldots, K_{N}$ of causal slices where $K_{j}$ has in-boundary $\Sigma_{\text {in }}^{j}$ and out-boundary $\Sigma_{\text {out }}^{j}$ such that $K^{j}$ and $K^{j+1}$ intersect in $\Sigma_{\text {out }}^{j}=\Sigma_{\text {in }}^{j+1}$ for $j=1,2 \ldots, N-1$, and $K^{i}$ and $K^{j}$ are disjoint otherwise. Then $K$ is a triangulation of $S^{2} \times[0,1]$ with boundary $\partial K=\Sigma_{\text {in }}^{1} \cup \Sigma_{\text {out }}^{N} \equiv \Sigma_{\text {in }} \cup \Sigma_{\text {out }}$.

If $T$ is a triangulation of the 3-dimensional ball with one interior vertex and boundary $\partial T=\Sigma_{\text {in }}$ then we obtain another triangulation of the ball by attaching $T$ to the causal triangulation $K$ along the in-boundary. Such triangulations are often referred to as causal triangulations of the 3-ball. Obviously we can close up at the other end in the same way and obtain a triangulation of $S^{3}$.

One can think of the triangulated 2-spheres in the boundaries of the causal slices as the "space" and the graph distance from $\Sigma_{\text {in }}$ as a discrete "time" coordinate so space-time is in this picture foliated by a sequence of 2-spheres which are connected by causal slices. This approach was introduced in [3] for 2-dimensions and generalized to 3-dimensions in [4]. For a while it was hoped that this model could be solved exactly in 3 dimensions because it can be mapped onto a certain matrix model [5] but this turned out to be overly optimistic. The 4-dimensional models are still under active investigation [6] but so far almost all results are numerical.

For the causal triangulations to make sense in 3 dimensions as a model for quantum gravity an exponential bound of the form (1) is also needed. The main purpose of this paper is to provide such a bound. Our method is partly inspired by the matrix model approach [5] which codes the information about the causal slices in certain graphs whose structure will be described in the next section.

We introduce the notion of generalized causal slices which are defined in the same way as causal slices except they are not required to be homeomorphic to $S^{2} \times[0,1]$. Instead they are required to be simplicial manifolds with two boundary components and this definition extends in a straightforward way to higher dimensions. Generalized causal triangulations are then defined as a sequence of generalized causal slices with boundaries identified as described above.

The principal results of this paper are the following: 
A The number $N_{3}(V)$ of combinatorially distinct 3-dimensional causal triangulations made up of $V$ tetrahedra satisfies the inequality (1).

B The two boundary components of generalized 3-dimensional causal triangulations are necessarily homeomorphic to each other and there is an exponential bound like (1) on the number of distinct generalized causal triangulations with boundaries of a fixed genus.

C Assuming the bound (1) for arbitrary triangulations of $S^{3}$, it is proven that the number of distinct causal triangulations of $S^{3} \times[0,1]$ is bounded by an exponential function of the number of 4-simplicies.

In the next section we establish some preliminary results on causal slices. In Section 3 we prove $\mathbf{A}$, discuss generalized causal triangulations and establish $\mathbf{B}$. It follows from $\mathbf{A}$, as we show in Section 3, that the number $N_{3}\left(V, \Sigma_{\text {in }}, \Sigma_{\text {out }}\right)$ of causal triangulations of $S^{2} \times[0,1]$ made up of $V$ tetrahedra with fixed boundaries $\Sigma_{\text {in }}$ and $\Sigma_{\text {out }}$ is given by

$$
N_{3}\left(V, \Sigma_{\text {in }}, \Sigma_{\text {out }}\right)=e^{\beta V+o(V)}
$$

where $\beta$ is independent of $\Sigma_{\text {in }}$ and $\Sigma_{\text {out. }}$. In Section 4 we extend some of the results from Section 3 to the 4-dimensional case and prove $\mathbf{C}$.

\section{Preliminaries}

In this section we establish some notation and give a general definition of the principal objects under study in this paper, causal slices and triangulations, as well as some associated objects.

Recall that an abstract simplicial complex $K$ is defined by its vertex set $K^{0}$, which here is assumed to be finite, and a set of subsets of $K^{0}$, called simplices, such that if $\sigma$ is a simplex in $K$ and $\sigma^{\prime} \subset \sigma$ then $\sigma^{\prime}$ is also a simplex in $K$ (see [15]). If $\sigma$ is a simplex in $K$ containing $p+1$ vertices it is called a $p$-simplex and the set of $p$-simplices will be denoted by $K^{p}$. We denote by $\left|K^{p}\right|$ the number of $p$-simplicies 
in $K$. If every simplex in $K$ is contained in some $D$-simplex we say that $D$ is the dimension of $K$. Given two abstract simplicial complexes $K$ and $K_{1}$, a bijective map $\psi: K_{1}^{0} \rightarrow K^{0}$ is called a combinatorial isomorphism if it induces a bijection of simplices. Obviously, combinatorial isomorphism is an equivalence relation.

A (geometric) realization of an abstract simplicial complex $K$ is a map

$$
\phi: K^{0} \rightarrow \mathbb{R}^{n}
$$

for some $n$ such that $\phi(\sigma)$ is an affinely independent set for every simplex $\sigma$ in $K$ and

$$
\operatorname{conv} \phi(\sigma) \cap \operatorname{conv} \phi\left(\sigma^{\prime}\right)=\operatorname{conv} \phi\left(\sigma \cap \sigma^{\prime}\right)
$$

for all simplices $\sigma, \sigma^{\prime}$ in $K$. Here, $\operatorname{conv} \phi(\sigma)$ is the convex hull of the set $\phi(\sigma)$. Hence, the convex spans of the images of abstract simplices in $K$ define a simplicial complex in $\mathbb{R}^{n}$ that we shall denote $K_{\phi}$. It is well known (see, e.g., [16]) that any abstract simplicial complex has a geometric realization for $n$ sufficiently large and that the homeomorphism class of

$$
K_{\phi}^{\prime}=\bigcup_{\sigma \in K} \operatorname{conv} \phi(\sigma)
$$

does not depend on the realization $\phi$. If $K_{\phi}^{\prime}$ is a manifold we call $K$ a manifold. In the following we assume that all manifolds are connected. Note also that if $\psi$ is a combinatorial isomorphism as defined above and $\phi$ is a realization of $K$ then $\phi \circ \psi$ is a realization of $K_{1}$ and we may speak about $\phi$ as a realization of the combinatorial isomorphism class of $K$. Frequently, we shall not distinguish between $K$, its combinatorial isomorphism class, and its geometric realizations. Similarly, if $K$ is a $D$-dimensional manifold, the boundary complex $\partial K$ is defined in the standard manner as an abstract simplicial complex consisting of all the $(D-1)$-simplices (together with their subsimplices) contained in only one $D$-simplex. A realization of $K$ gives in an obvious way rise to a realization of $\partial K$ in the same Euclidean space.

From now on we shall assume that $K$ is a manifold of dimension $D$. For the purpose of defining the causal slices we further assume that $K^{0}$ is divided into two classes whose vertices we will call red and blue. These will be the vertices of the 

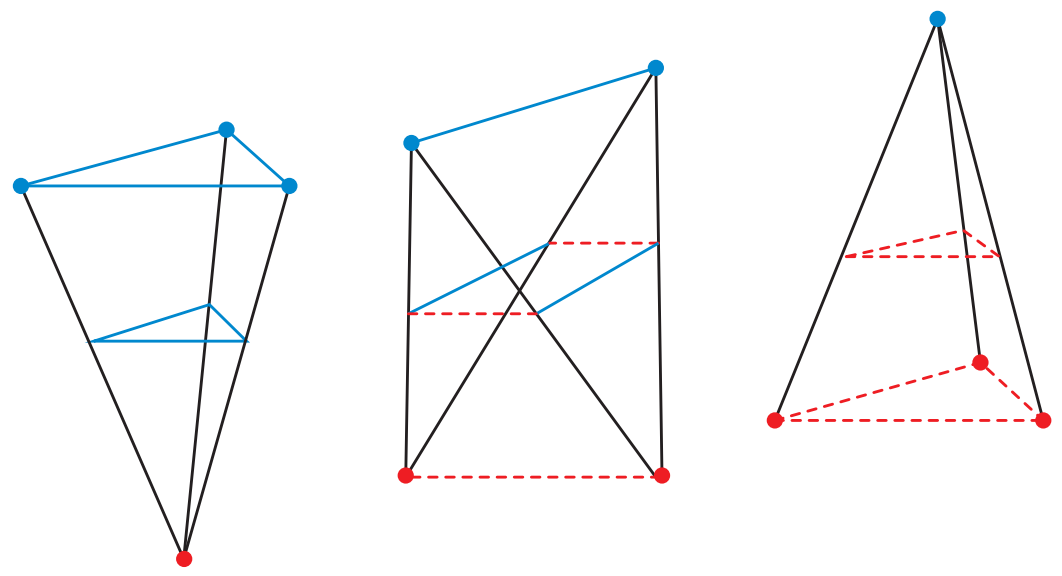

Figure 1: The three different types of $D$-simplicies that arise for $D=3$. The full lines are blue and the broken ones red.

in- and out-boundary components discussed in the Introduction. By definition, a $p$-simplex whose vertices all have the same colour inherits the colour of the vertices. We will require that no $D$-simplex has all its vertices of the same colour. The $D$ simplices therefore fall into $D$ classes determined by the number $k$ of red vertices, and hence $D+1-k$ blue vertices, which we say are of type $(k, D+1-k)$, see Fig. 1.

Definition 1. A D-dimensional causal slice $K$ is an abstract simplicial complex as described above which satisfies the following conditions:

(i) $K$ is homeomorphic to the cylinder $S^{D-1} \times[0,1]$.

(ii) All mono-coloured simplices of $K$ belong to the boundary $\partial K$, such that the red ones belong to one boundary component $\partial K_{\text {red }}$ and the blue ones to the other component $\partial K_{\text {blue }}$.

A causal triangulation of dimension $D$ is an abstract simplicial complex of the form

$$
M=\bigcup_{i=1}^{N} K^{i},
$$


where each $K^{i}$ is a causal slice of dimension $D$ such that $K^{i}$ is disjoint from $K^{j}$ for $i \neq j$ except that $\partial K_{b l u e}^{i}=\partial K_{\text {red }}^{i+1}, i=1, \ldots, N-1$, as uncoloured abstract simplicial complexes. The boundary components of $\partial K_{\text {red }}^{1}$ and $\partial K_{b l u e}^{N}$ of $M$ will be denoted $\Sigma_{\text {in }}$ and $\Sigma_{\text {out }}$, respectively.

With the colouring conventions described above, we note that (ii) in Definition 1 is equivalent to the requirement in the Introduction (where we had $D=3$ ) that all vertices belong to the boundary and no tetrahedron has all its vertices in the same boundary component. There is an obvious notion of combinatorial isomorphism of causal slices respecting the colouring, and we shall denote by $\mathcal{C S}_{D}$ the set of combinatorial equivalence classes of $D$-dimensional causal slices. Similarly, we denote by $\mathcal{C}_{D}$ the set of combinatorial equivalence classes of causal triangulations of dimension $D$.

Let $K$ be a causal slice of dimension $D$ and consider a realization $\phi$ of $K$ in $\mathbb{R}^{n}$. Denote the boundary components of $K_{\phi}^{\prime}$ corresponding to $\partial K_{\text {red }}$ and $\partial K_{\text {blue }}$ by $\partial K_{\text {red }}^{\prime}$ and $\partial K_{\text {blue }}^{\prime}$, respectively. Given $x \in K_{\phi}^{\prime}$ there is a $D$-simplex $s$ in $K_{\phi}$ containing $x$. If we let $\left\{r_{i}\right\}$ and $\left\{b_{j}\right\}$ denote the red and blue vertices, respectively, in $s$, we can express $x$ in a unique way as a convex combination

$$
x=\sum_{i} \mu_{i} r_{i}+\sum_{j} \lambda_{j} b_{j},
$$

where $\mu_{i}, \lambda_{j} \geq 0$ and $\sum_{i} \mu_{i}+\sum_{j} \lambda_{j}=1$. Depending on the colour type of $s, i$ runs from 1 to $k$ and $j$ runs from 1 to $D+1-k, 1 \leq k \leq D$. We define the height function $h: K_{\phi}^{\prime} \rightarrow[0,1]$ by

$$
h(x)=\sum_{j} \lambda_{j}
$$

It is easy to check that this function is well defined, i.e., $h(x)$ does not depend on the choice of $s$ containing $x$, and it is a simplicial map, see [15]. Evidently, $h(x)=0$ if and only if $x \in \partial K_{\text {red }}^{\prime}$ and $h(x)=1$ if and only if $x \in \partial K_{b l u e}^{\prime}$. Moreover, if $0<h(x)<1$, then there is a unique line segment $\left[x_{r}, x_{b}\right]$ with endpoints $x_{r} \in \partial K_{r e d}^{\prime}$ and $x_{b} \in \partial K_{b l u e}^{\prime}$ that contains $x$ and is contained in some $D$-simplex in $K_{\phi}^{\prime}$. With 
the notation introduced above we have

$$
x_{r}=\frac{1}{1-h(x)} \sum_{i} \mu_{i} r_{i}, \quad x_{b}=\frac{1}{h(x)} \sum_{j} \lambda_{j} b_{j} .
$$

We now consider the section in $K_{\phi}^{\prime}$ consisting of points at height $\frac{1}{2}$ :

$$
S_{K}=\left\{x \in K_{\phi}^{\prime}: h(x)=\frac{1}{2}\right\}
$$

We will refer to $S_{K}$ as the midsection of $K_{\phi}^{\prime}$. Actually, we shall consider $S_{K}$ as a coloured cell complex in the following sense, that we now describe in detail only for the cases $D=3$ and $D=4$. We refer to [15] for a definition of cell complexes.

Consider first the case $D=3$. If $s$ is a tetrahedron in $K_{\phi}$ of type $(k, 4-k)$, $k \in\{1,2,3\}$, then $s \cap S_{K}$ is a cell with $k(4-k)$ corners. If $k=1$ or 3 , then the cell is a triangle whose edges we declare to be red if $k=3$ but blue if $k=1$. If $k=2$ then the cell $s \cap S_{K}$ is a quadrangle, i.e., a product of two 1-simplicies. The edges of the quadrangles are coloured by the same colour as the colour of the boundary edge in $\partial K_{\text {red }}^{\prime}$ or $\partial K_{\text {blue }}^{\prime}$ of the triangle to which they belong. In particular, opposite sides of the quadrangles have the same colour, see Fig. 2.

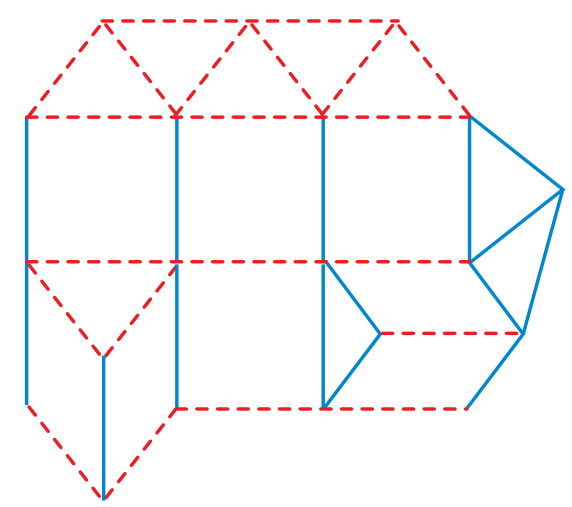

Figure 2: A part of a midsection of a 3-dimensional causal slice. 
This defines $S_{K}$ as a 2-dimensional cell complex with coloured edges. We shall also use $S_{K}$ to denote its combinatorial isomorphism class, where a combinatorial (or abstract in the terminology of [15]) isomorphism $\varphi: S_{K_{1}} \rightarrow S_{K_{2}}$ is defined as a bijective map from the 0-cells of $S_{K_{1}}$ onto those of $S_{K_{2}}$ such that $\left\{v_{1}, \ldots, v_{k}\right\}$ is the set of corners of a cell in $S_{K_{1}}$ if and only if $\left\{\varphi\left(v_{1}\right), \ldots, \varphi\left(v_{k}\right)\right\}$ is the set of corners of a cell in $S_{K_{2}}$ for $k=2,3,4$ and, in addition, $\varphi$ preserves the colouring of edges. Note that changing the hight of the midsection to any $h \in(0,1)$ does not affect its combinatorial equivalence class but at $h=0$ and $h=1$ the section collapses to the red and blue boundaries, respectively.

In case $D=4$ there are four types of coloured cells. If $s$ is a 4 -simplex of type $(4,1)$ or $(1,4)$ then $s \cap S_{K}$ is a tetrahedron coloured red or blue, respectively. On the other hand, if $s$ is of type $(3,2)$ or $(2,3)$ then $s \cap S_{K}$ is a prism $\Delta \times[0,1]$, where $\Delta$ is a triangle. In the former case, the edges of the two triangles in the boundary are coloured red while the three remaining edges are coloured blue, and vice versa in the latter case (see Fig. 3). This defines $S_{K}$ as a 3-dimensional cell complex with coloured edges.
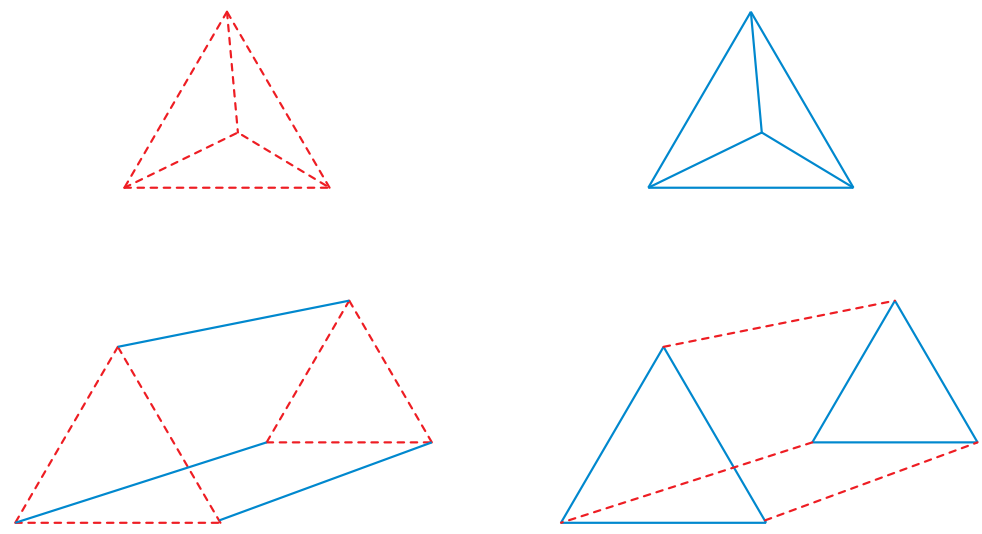

Figure 3: The four different coloured cells that occur in the midsection in the 4dimensional case. 
While we need a realization of $K$ in order to define the midsection, it is essentially independent of which realization is chosen as decribed in the following Lemma. This justifies the notation $S_{K}$ for the midsection.

Lemma 1. The midsection $S_{K}$ of a causal slice $K$ is a closed $(D-1)$-dimensional manifold. Combinatorially isomorphic causal slices give rise to combinatorially isomorphic midsections.

Proof. Suppose we have a given realization $K_{\phi}$ of $K$ with a midsection $S_{K}$. We have that $\frac{1}{2}$ is a regular value of the height function $h$, from which it follows that $S_{K}$ is a closed $(D-1)$-dimensional manifold (see, e.g., [18] Sections 1.3 and 4.2).

If $\psi$ is a combinatorial isomorphism between abstract simplicial complexes $K_{1}$ and $K_{2}$ there is a canonical piecewise linear homeomorphism between any two realizations $K_{\phi_{1}}$ and $K_{\phi_{2}}$ of them, which obviously restricts to a piecewise linear homeomorphism between the corresponding midsections $S_{K_{\phi_{1}}}$ and $S_{K_{\phi_{2}}}$ which in turn induces a combinatorial isomorphism between $S_{K_{\phi_{1}}}$ and $S_{K_{\phi_{2}}}$. This proves the last statement of the Lemma.

The preceding Lemma allows us to define a mapping $\pi$ on $\mathcal{C} \mathcal{S}_{D}$ by setting

$$
\pi(K)=S_{K},
$$

such that $\pi$ takes values in the set of $(D-1)$-dimensional closed coloured cellcomplexes as described above for the cases $D=3$ and $D=4$. Straightforward generalizations can be given for higher dimensions but we shall not consider these cases further in this paper. It is rather easy to see that $\pi$ is not a surjective map, see Fig. 4.

More specifically, there exist coloured cell complexes as we have described that contain vertices which are connected by a red path, i.e., a path consisting entirely of red edges, as well as by a blue path and such complexes cannot be the midsection of a causal slice. To see this, suppose $v$ and $w$ are vertices connected by a blue path. Then $v$ and $w$ lie on two unique 1-simplicies with the same red endpoint in the boundary of the slice. If they are also connected by a red path those two 1-simplices 


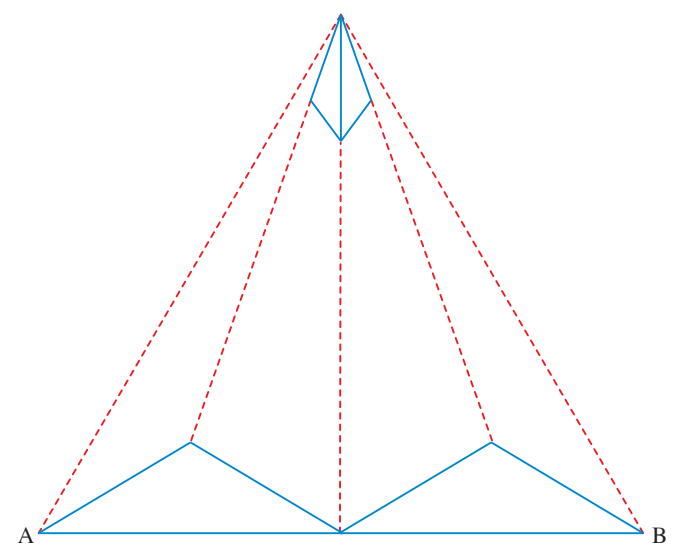

Figure 4: Taking two copies of this cell complex which has the topology of a disc and identifying the boundary edges of the same color we obtain a coloured cell complex with the topology of $S^{2}$. The vertices $A$ and $B$ are connected both by a red and a blue path.

have the same blue endpoint in the boundary. This is only possible if $v=w$. It is beyond the scope of the present paper to characterize those $(D-1)$-dimensional cell complexes that arise as midsections of causal slices. On the other hand, it will be an important ingredient in the proof of the exponential bound to show that $\pi$ is injective.

Before proceeding it is useful to introduce the following notation. If $\sigma_{1}, \ldots, \sigma_{n}$, are simplices whose vertices form a partition of the vertices of a simplex $\sigma$ we use the notation $\left(\sigma_{1} \ldots \sigma_{n}\right)$ for $\sigma$. Thus, e.g., if $v$ is a vertex and $e$ is an edge then (ve) denotes the triangle containing the edge $e$ and opposite vertex $v$.

If $K_{\phi}$ is a geometric realization of an abstract simplicial complex $K$ in $\mathbb{R}^{n}$ and $\sigma$ is a simplex in $K$ we let $N_{\epsilon}(\sigma)$ denote the neighbourhood of $\phi(\sigma)$ consisting of points in $K_{\phi}^{\prime}$ at distance at most $\epsilon$ from $\phi(\sigma)$. We shall repeatedly use the fact that if $K$ is a connected manifold of dimension $D$ and $S$ is a union of neighbourhoods of the form $N_{\epsilon}(\sigma)$ with $\sigma$ of dimension at most $D-2$ then $K_{\phi}^{\prime} \backslash S$ is connected if $\epsilon>0$ is sufficiently small. Likewise, we will use the fact that the star of a simplex not in the boundary of $K$ is homeomorphic to a $D$-ball and, in particular, that any 
interior $(D-1)$-simplex is incident on exactly two $D$-simplices.

\section{The 3-dimensional case}

In this section we first establish an exponential bound on the number of 3-dimensional causal triangulations introduced in Definition 1. We then generalize this result to arbitrary boundary topology and discuss a slightly generalized notion of causal triangulations.

Theorem 1. Let $N_{3}(V)$ denote the number of 3-dimensional causal triangulations with $V$ tetrahedra. Then there exists a constant $C_{3}>0$ such that

$$
N_{3}(V) \leq C_{3}^{V}
$$

for all positive integers $V$.

Proof. The proof proceeds in three steps.

(i) Since the number of ways to write $V$ as a sum of positive integers is bounded by $2^{V}$ it suffices to find an exponential bound on the number of causal slices as a function of the number $V$ of tetrahedra.

(ii) Consider a causal slice $K$. By assumption its interior is homeomorphic to $S^{2} \times(0,1)$. On the other hand, we have already remarked that it is also homeomorphic to $S_{K} \times(0,1)$ since the topology of the midsection does not depend on its height $h \in(0,1)$. In particular, $S_{K} \times(0,1)$ is simply connected and it follows that $S_{K}$ is simply connected and hence homeomorphic to $S^{2}$. Now introduce a new edge for each quadrangle in $S_{K}$ connecting an opposite pair of its vertices and colour those edges black. This yields an abstract triangulation of $S^{2}$ with at most $2 \mathrm{~V}$ triangles, since each rectangle in $S_{K}$ splits into two triangles. Clearly, removing the black edges one recovers $S_{K}$ and the number of possible edge colourings is bounded by $3^{3 V}$, since the number of edges in a triangulation of $S^{2}$ with $N$ triangles equals $\frac{3}{2} N$. Moreover, it is well known that the number of (uncoloured) abstract triangulations of $S^{2}$ with at most $N$ triangles is bounded by $C_{0}^{N}$, where $C_{0}>0$ is a constant 
(see, e.g., [11] for an elementary proof; one may also use the corresponding result for planar maps [17]). We conclude that the number of combinatorially distinct midsections $S_{K}$, that can occur for $\left|K^{3}\right|=V$, is bounded by $\left(27 C_{0}^{2}\right)^{V}$.

(iii) If $S_{K}$ determines $K$ up to combinatorial isomorphism, that is if the map $\pi$ is injective on $\mathcal{C S}_{3}$, then the bound (7) follows from (i) and (ii). The proof of injectivity of $\pi$ is deferred to Lemma 2 below.

Lemma 2. Let $K \in \mathcal{C S}_{3}$. Then the coloured cell complex $S_{K}$ determines $K$ up to combinatorial isomorphism.

Proof. Let $K$ be a causal slice with realization $K_{\phi}$ containing $S_{K}$. We will construct from $S=S_{K}$ a simplicial complex $K_{S}$, depending only on the combinatorial isomorphism class of $S_{K}$, which will be shown to be isomorphic to $K$.

To each vertex $v$ in $S_{K}$ we associate a pair of new vertices $\left(r_{v}, b_{v}\right)$ which will be in the vertex set of $K_{S}$. The vertex $r_{v}$ is by assumption different from $b_{w}$ for all vertices $v, w$ in $S_{K}$ and $r_{v}=r_{w}$ if and only if $v$ is connected to $w$ in $S_{K}$ by a blue path. Similarly, $b_{v}=b_{w}$ if and only if $v$ is connected to $w$ by a red path. We note that $r_{v_{1}}=r_{v_{2}}$ and $b_{v_{1}}=b_{v_{2}}$ if and only if $v_{1}=v_{2}$, since the edges in $K_{\phi}$ containing $v_{1}$ and $v_{2}$ would have identical endpoints. The vertex set $K_{S}^{0}$ of $K_{S}$ is by definition the set of vertices $r_{v}, b_{v}$ where $v$ ranges over the vertices of $S_{K}$, subject to the identifications we have described.

Now associate to each red triangle of $S_{K}$ with corners $v_{1}, v_{2}, v_{3}$ a tetrahedron of type $(3,1)$ with red corners $r_{v_{1}}, r_{v_{2}}, r_{v_{3}}$ and a blue corner $b_{v_{1}}=b_{v_{2}}=b_{v_{3}}$. Analogously we associate a type $(1,3)$ tetrahedron to each blue triangle in $S$. Finally, to a quadrangle cell with corners $v_{1}, v_{2}, v_{3}, v_{4}$ such that $\left(v_{1}, v_{2}\right)$ and $\left(v_{3}, v_{4}\right)$ are red edges while $\left(v_{2}, v_{3}\right)$ and $\left(v_{4}, v_{1}\right)$ are blue, we associate a type $(2,2)$ tetrahedron with red corners $r_{v_{1}}=r_{v_{4}}$ and $r_{v_{2}}=r_{v_{3}}$ and blue corners $b_{v_{1}}=b_{v_{2}}$ and $b_{v_{3}}=b_{v_{4}}$. This defines the set of tetrahedra in $K_{S}$ and hence also defines $K_{S}$ as a 3-dimensional abstract simplicial complex with obvious vertex colouring.

We next remark that if $v_{1}, v_{2}$ are two vertices in $S_{K}$ connected by a blue path 
then, as noted above, the unique edges in $K_{\phi}$ containing $v_{1}$ and $v_{2}$, respectively, have a common red endpoint which will be denoted $\bar{r}\left(v_{1}\right)=\bar{r}\left(v_{2}\right)$. Similarly, if $v_{1}, v_{2}$ are connected by a red path the two edges have a common blue endpoint $\bar{b}\left(v_{1}\right)=\bar{b}\left(v_{2}\right)$. Hence, we may define a map $\tau: K_{S}^{0} \rightarrow K^{0}$ by setting

$$
\tau\left(r_{v}\right)=\bar{r}(v) \quad \text { and } \quad \tau\left(b_{v}\right)=\bar{b}(v) .
$$

It is evident by construction of $K_{S}$ that $\tau$ is surjective and maps simplices in $K_{S}$ to simplices in $K$. To prove that $\tau$ is a combinatorial isomorhism, it remains to show that $\tau$ is injective, i.e., that the defining identifications of vertices described above are the only ones.

In order to prove this consider, say, a red vertex $r$ and the star $B_{r}$ in $\partial K_{\text {red }}$ around $r$. It is a disc whose boundary $\partial B_{r}$ is a circle $S^{1}$ consisting of red edges $e_{1}, \ldots, e_{n}$, see Fig. 5 . The boundary triangle $\Delta_{i}=\left(r e_{i}\right)$ is incident on exactly one tetrahedron

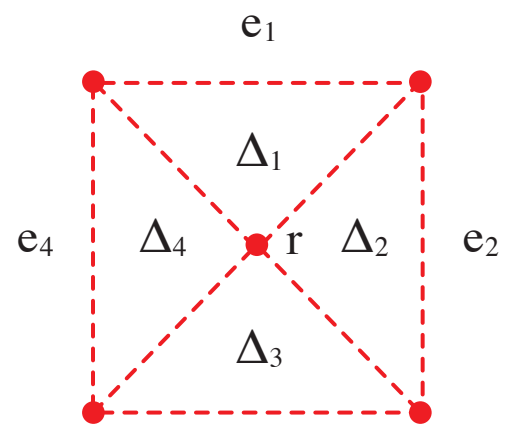

$\mathrm{e}_{3}$

Figure 5: The star in $\partial K_{\text {red }}$ of a red boundary vertex $r$.

$t_{i}=\left(r b_{i} e_{i}\right)$ which is necessarly of type $(3,1)$, as indicated, with $b_{i}$ denoting its blue vertex. Consider a vertex $v \in \partial B_{r}$. We note that $r$ is not contained in any simplex of type $(3,1)$ other than $t_{1}, \ldots, t_{n}$, since there are no mono-coloured interior edges in $K$. The boundary edge $(r v)$ is contained in exactly two triangles $\Delta_{i}$ and $\Delta_{i+1}$ in $B_{r}$. If $b_{i} \neq b_{i+1}$ the triangle $\left(r v b_{i}\right)$ is incident on $t_{i}$ and one other tetrahedron 
$t_{i 1}=\left(r v b_{i} b_{i 1}\right)$, necessarily of type $(2,2)$. If $b_{i 1} \neq b_{i+1}$ the triangle $\left(r v b_{i 1}\right)$ is incident on $t_{i 1}$ and one other tetrahedron $t_{i 2}=\left(r v b_{i 1} b_{i 2}\right)$, necessarily of type $(2,2)$. Continuing, we obtain a sequence $t_{i j}=\left(r v b_{i(j-1)} b_{i j}\right), j=1, \ldots, m_{i}$, of tetrahedra of type $(2,2)$ such that $b_{i 0}=b_{i}$ and $b_{i m_{i}}=b_{i+1}$. These are by construction different tetrahedra, since $b_{i 0}, \ldots, b_{i m_{i}}$ are different. If $b_{i}=b_{i+1}$ we set $m_{i}=0$, in which case the triangle $\left(r v b_{i}\right)$ is incident only on the tetrahedra $t_{i}$ and $t_{i+1}$. It is important to note that the edge $(r v)$ is not incident on any tetrahedra in $K$ except $t_{i}, t_{i 1}, \ldots, t_{i m_{i}}, t_{i+1}$, since any other tetrahedron incident on $(r v)$ would intersect $t_{i} \cup t_{i 1} \cup \cdots \cup t_{i m_{i}} \cup t_{i+1}$ only in $(r v)$ and it would follow that removing a neighbourhood $N_{\epsilon}(r v)$ from the star of $(r v)$ would yield a disconnected set for $\epsilon$ small enough, contradicting the manifold property of $K$.

Repeating the above construction for all vertices $v \in \partial B_{r}$ we obtain a connected simplicial complex $A_{0}$ consisting of tetrahedra $t_{i}, t_{i 1}, \ldots, t_{i m_{i}}, i=1, \ldots, n$, and their subsimplices. From the preceding remark it follows that $A_{0}$ exhausts all the tetrahedra of type $(3,1)$ or $(2,2)$ in $K$ that are incident on $r$. We next describe how the star of $r$ in $K$ is obtained by successively adding tetrahedra, necessarily of type $(1,3)$, starting from $A_{0}$.

Let $C_{0}$ be the connected simplicial complex consisting of the blue edges and vertices of $A_{0}$, i.e., the edges of $C_{0}$ are $e_{i j}=\left(b_{i(j-1)} b_{i j}\right), 1 \leq j \leq m_{i}, 1 \leq i \leq n$, some of which may be identical. Pick an edge $e_{i j}$ in $C_{0}$. The triangle $\Delta_{i j}=\left(r e_{i j}\right)$ is contained in $t_{i j}$ and one more tetrahedron $t$ in $K$. If $t$ is not in $A_{0}$ it is of type $(1,3)$ and we define $A_{1}$ to be the simplicial complex obtained by adding $t$ (and its subsimplices) to $A_{0}$. Moreover, $C_{1}$ is defined by adding the triangle in $t$ opposite to $r$ to $C_{0}$. If $t$ is already in $A_{0}$ we set $A_{1}=A_{0}$ and $C_{1}=C_{0}$. Next repeat the same construction for any edge $e$ in $C_{1}$ to obtain $A_{2}$ and $C_{2}$ and continue until the complex $A_{N}$ and its blue subcomplex $C_{N}$ are obtained with the property that any triangle $(r e)$, where $e$ is an edge in $C_{N}$, is incident on exactly two tetrahedra in $A_{N}$.

Suppose there exists a tetrahedron $t^{\prime}$ of type $(1,3)$ incident on $r$ which is not in $A_{N}$. Then $t^{\prime}$ is not incident on any triangle in $A_{N}$ by construction and hence 
it can share at most two edges with $A_{N}$. It follows that by removing a sufficiently small neighbourhood of the 1-skeleton of the star of $r$ in $K$ one would obtain a disconnected set, contradicting the manifold property of $K$. Thus we have shown that $A_{N}$ equals the star $\widetilde{B}_{r}$ of $r$ in $K$. Moreover, the blue subcomplex $C_{N}$ of $A_{N}$ is by construction connected and its vertices are exactly the set of blue vertices that occur as endpoints of edges in $K$ whose other endpoint is $r$. It follows that any two midpoints of those edges in $S_{K}$ are connected by a blue path. Applying an analogous argument for the blue vertices $b \in \partial K_{\text {blue }}$, we conclude that $\tau$ is injective and this completes the proof that $\tau$ is a combinatorial isomorphism.

It is clear from the construction of $K_{S}$ that it depends only on the combinatorial isomorphism class of $S=S_{K}$. It therefore follows from the existence of $\tau$ that $S_{K}$ determines $K$ up to combinatorial isomorphism as claimed.

Remark. An alternative route to Theorem 1 would be to show that every causal slice has a local construction in the sense of [12]. While we believe that this is feasible, we find the argument using the midsection $S_{K}$ more transparent.

The exponential bound in Theorem 1 and some standard subadditivity arguments allow us to get a sharper estimate on the growth of the number of 3-dimensional causal triangulations with fixed boundaries as we now explain. Let $\mathcal{T}\left(\Sigma_{\text {in }}, \Sigma_{\text {out }}\right)$ be the set of all (combinatorial isomorphism classes of) 3-dimensional causal triangulations with boundary components $\Sigma_{\text {in }}$ and $\Sigma_{\text {out }}$. Let $N\left(V, \Sigma_{\text {in }}, \Sigma_{\text {out }}\right)$ denote the number of triangulations in $\mathcal{T}\left(\Sigma_{\text {in }}, \Sigma_{\text {out }}\right)$ of volume $V$, i.e., consisting of $V$ tetrahedra.

Theorem 2. The limit

$$
\lim _{V \rightarrow \infty} \frac{\log N\left(V, \Sigma_{\text {in }}, \Sigma_{\text {out }}\right)}{V}
$$

exists for all $\Sigma_{\mathrm{in}}, \Sigma_{\mathrm{out}}$ and is independent of $\Sigma_{\mathrm{in}}$ and $\Sigma_{\mathrm{out}}$.

In order to prove this Theorem we need the following simple Lemma which we assume for a moment. 
Lemma 3. The sets $\mathcal{T}\left(\Sigma_{\mathrm{in}}, \Sigma_{\text {out }}\right)$ are all nonempty.

Proof of Theorem 2. Let $\Sigma_{\text {in }}$ and $\Sigma_{\text {out }}$ be two triangulations of $S^{2}$. Choose a causal triangulation $T_{0} \in \mathcal{T}\left(\Sigma_{\text {in }}, \Sigma_{\text {out }}\right)$ and let $V_{0}$ be the volume of $T_{0}$. Let $T_{1}$ and $T_{2}$ be two causal triangulations in the same set with volumes $V_{1}$ and $V_{2}$. Now glue $T_{1}$ to $T_{0}$ along $\Sigma_{\text {out }}$ and glue $T_{2}$ to $T_{0}$ along $\Sigma_{\text {in }}$, using arbitrarily chosen combinatorial isomorphisms to identify boundary components. Then we obtain a new element $T^{\prime}$ in $\mathcal{T}\left(\Sigma_{\text {in }}, \Sigma_{\text {out }}\right)$ of volume $V_{1}+V_{2}+V_{0}$. Clearly $T_{1}$ and $T_{2}$ are uniquely determined by $T^{\prime}$ and it follows that

$$
N\left(V_{1}, \Sigma_{\text {in }}, \Sigma_{\text {out }}\right) N\left(V_{2}, \Sigma_{\text {in }}, \Sigma_{\text {out }}\right) \leq N\left(V_{1}+V_{2}+V_{0}, \Sigma_{\text {in }}, \Sigma_{\text {out }}\right)
$$

Hence, the function

$$
f(V)=-\log N\left(V-V_{0}, \Sigma_{\text {in }}, \Sigma_{\text {out }}\right)
$$

is subadditive (for $V$ sufficiently large) so (see, e.g., [13] Sec. 7.6)

$$
-\lim _{V \rightarrow \infty} \frac{f(V)}{V}=-\inf _{V} \frac{f(V)}{V} \equiv \beta\left(\Sigma_{\text {in }}, \Sigma_{\text {out }}\right)
$$

which is finite by Theorem 1. A priori $\beta\left(\Sigma_{\text {in }}, \Sigma_{\text {out }}\right)$ depends on the boundary triangulations $\Sigma_{\text {in }}$ and $\Sigma_{\text {out }}$. However, given an arbitrary causal triangulation in $\mathcal{T}\left(\Sigma_{\text {in }}, \Sigma_{\text {out }}\right)$ we can glue on it two fixed triangulations $T_{1}$ and $T_{2}$ from $\mathcal{T}\left(\Sigma_{\text {in }}^{\prime}, \Sigma_{\text {in }}\right)$ and $\mathcal{T}\left(\Sigma_{\text {out }}, \Sigma_{\text {out }}^{\prime}\right)$ for any triangulations $\Sigma_{\text {in }}^{\prime}$ and $\Sigma_{\text {out }}^{\prime}$ of the 2 -sphere. It follows that

$$
N\left(V, \Sigma_{\text {in }}, \Sigma_{\text {out }}\right) \leq N\left(V+V_{1}+V_{2}, \Sigma_{\text {in }}^{\prime}, \Sigma_{\text {out }}^{\prime}\right),
$$

where $V_{i}$ is the volume of $T_{i}, i=1,2$. We deduce that

$$
\beta\left(\Sigma_{\text {in }}, \Sigma_{\text {out }}\right) \leq \beta\left(\Sigma_{\text {in }}^{\prime}, \Sigma_{\text {out }}^{\prime}\right)
$$

for any two pairs of triangulations of $S^{2}$. Hence, all the $\beta$ 's are equal and we have established the Theorem.

Proof of Lemma 3. Let $\Sigma_{T}$ be the boundary of a tetrahedron. We will show that for any triangulation $\Sigma$ of the 2-sphere there is a causal slice with boundary components $\Sigma$ and $\Sigma_{T}$. Gluing two such causal slices along the boundary tetrahedron we can then obtain a causal triangulation with any prescribed in- and out-boundaries. 
Consider the cone $C_{\Sigma}$ over $\Sigma$, that is to say the 3-dimensional simplicial complex constructed by introducing one new vertex $a$ and declaring the tetrahedra to be $(\Delta a)$ where $\Delta$ ranges over the triangles in $\Sigma$. The tetrahedra $(\Delta a)$ and $\left(\Delta^{\prime} a\right)$ are glued together along the triangle $(\ell a)$ if and only if the two triangles $\Delta$ and $\Delta^{\prime}$ in $\Sigma$ are glued along the edge $\ell$.

Now suppose for simplicity that $\Sigma$ has a vertex 0 of degree three. Denote the neighbours of 0 by 1,2 and 3. Consider the star of the vertex 0 in the cone $C_{\Sigma}$ which consists of the tetrahedra (012a), (013a) and (023a). Remove these three tetrahedra from $C_{\Sigma}$. Take a copy of $\Sigma_{T}$ and identify one of the vertices with $a$. Label the other vertices $b, c, d$. Colour the vertices and edges in $\Sigma$ red and those in $\Sigma_{T}$ blue. Now we fill up this simplicial complex so that we get a causal slice. First introduce the $(3,1)$ tetrahedra (b023), (c013) and (d012) which fill the hole in $\Sigma$. Then we glue $(1,3)$ tetrahedra to the $\Sigma_{T}$. These are: $(a c d 1),(a b d 2),(a b c 3)$ and $(b c d 0)$. It remains to glue the $(1,3)$ tetrahedra to the $(3,1)$ tetrahedra. For this purpose we need $6(2,2)$ tetrahedra which are (cd01), (bd02), (bc03), (ac13), (ad12) and (ab23), see Fig. 6 which illustrates part of the midsection of the causal slice we have constructed.

In this construction we began by removing 3 tetrahedra from $C_{\Sigma}$ and then we added 13 tetrahedra to get a causal slice with boundary components $\Sigma_{T}$ and $\Sigma$. Hence, for any two triangulations $\Sigma_{\text {in }}$ and $\Sigma_{\text {out }}$ of $S^{2}$ there is a causal triangulation of volume $\left|\Sigma_{\text {in }}\right|+\left|\Sigma_{\text {out }}\right|+20$ with boundary components $\Sigma_{\text {in }}$ and $\Sigma_{\text {out }}$. Here $\left|\Sigma_{\text {in }}\right|$ is the number of triangles in $\Sigma_{\text {in }}$ and similarly for $\Sigma_{\text {out }}$.

If $\Sigma$ has no vertex of degree 3 , it necessarily has a vertex of order 4 or 5 . It is easy modify the construction above to this case by subdividing some of the tetrahedra. We leave the details to the reader.

In the remainder of this section we discuss 3-dimensional causal triangulations with boundary components of arbitrary topology. We also relax the requirement of cylindrical topology in condition (i) of Definition 1.

Definition 2. A D-dimensional generalized causal slice $K$ is an abstract simplicial complex which satisfies the following conditions: 


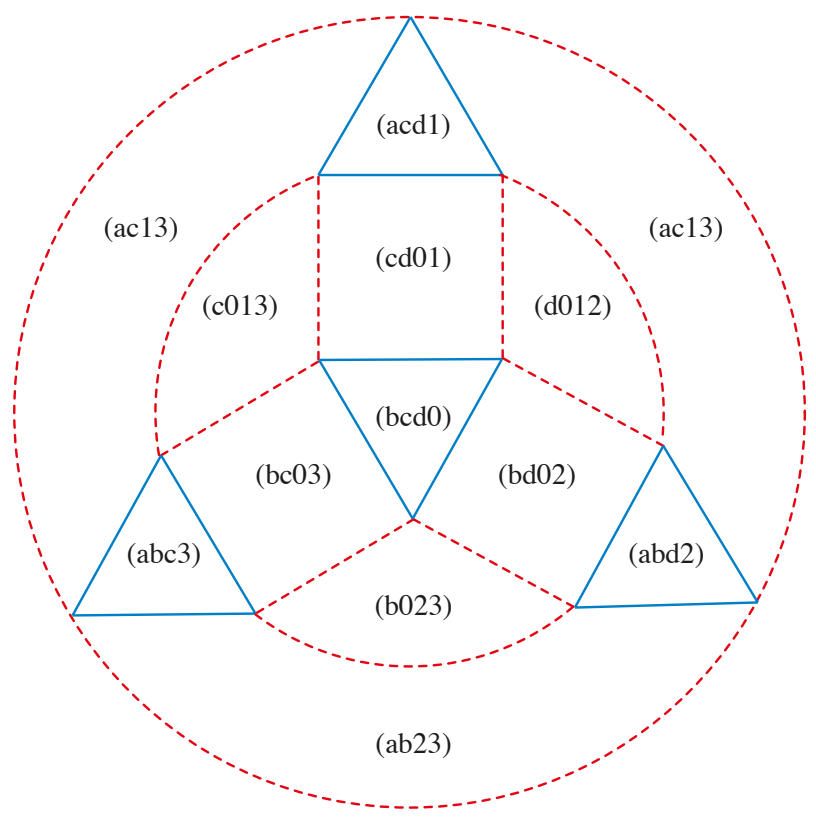

Figure 6: The part of the midsection describing the gluing of the 13 tetrahedra replacing the star of 0 in $C_{\Sigma}$. Here $(a c 13)$ refers to the tetrahedron with vertices $a, c, 1,3$ etc.

(i) $K$ is an oriented $D$-manifold with two boundary components.

(ii) All mono-coloured simplices of $K$ belong to the boundary, such that the red ones belong to one boundary component $\partial K_{\text {red }}$ and the blue ones to the other component $\partial K_{\text {blue }}$.

A generalized causal triangulation of dimension $D$ is an abstract simplicial complex of the form

$$
M=\bigcup_{i=1}^{N} K^{i},
$$

where each $K^{i}$ is a $D$-dimensional generalized causal slice such that $K^{i}$ and $K^{j}$ are disjoint if $i \neq j$ except that $\partial K_{\text {blue }}^{i}=\partial K_{\text {red }}^{i+1}, i=1, \ldots, N-1$, as uncoloured abstract simplicial complexes. The boundary components of $\partial K_{\text {red }}^{1}$ and $\partial K_{b l u e}^{N}$ of $K$ will be denoted $\Sigma_{\text {in }}$ and $\Sigma_{\text {out }}$, respectively. 
Lemma 4. For any generalized 3-dimensional causal slice $K$ the midsection $S_{K}$ is homeomorphic to both $\partial K_{\text {red }}$ and $\partial K_{\text {blue }}$.

Remark. Before proving the above lemma we note that if we assume that $K$ is a topological cylinder, i.e., homeomorphic to $[0,1] \times \Sigma$ for some two-dimensional orientable manifold $\Sigma$, then the statement of Lemma 4 is trivial since we know that the interior of $K$ is homeomorphic to $(0,1) \times S_{K}$ and hence the homotopy group $\pi_{0}\left(S_{K}\right)$ is isomorphic to $\pi_{0}(\Sigma)$ and this implies that $S_{K}$ is homeomorphic to $\Sigma$.

Proof. Let $K$ be a generalized causal slice. We use the same notation as in the proof of Lemma 2 and consider the star $\widetilde{B}_{r}$ in $K$ around a red vertex $r \in \partial K_{\text {red }}$ consisting of type $(3,1)$ tetrahedra $t_{i}$ and type $(2,2)$ tetrahedra $t_{i j}$ and possibly additional tetrahedra of type $(1,3)$.

Since $K$ is an oriented manifold it is clear that $S_{K}$ is an orientable surface. It therefore suffices to show that the Euler characteristics of $\partial K_{\text {red }}$ and $S_{K}$ coincide, the argument for $\partial K_{\text {blue }}$ being similar.

Consider the intersection of $\widetilde{B}_{r}$ with $S_{K}$. Its boundary $\partial\left(\widetilde{B}_{r} \cap S_{K}\right)$ in $S_{K}$ is a simple curve $\mu_{r}$ consisting of edges each of which belongs to either a quadrangle $t_{i j} \cap S_{K}$ and is blue or to a red triangle $t_{i} \cap S_{K}$. Since $\partial \widetilde{B}_{r} \backslash \partial K_{\text {red }}$ is a disc, it follows by the manifold property of $K$ that $\mu_{r}$ bounds a disc in $\partial \widetilde{B}_{r} \backslash \partial K_{\text {red }}$ and this implies that $\widetilde{B}_{r} \cap S_{K}$ is a disc containing the quadrangles $t_{i j} \cap S_{K}$ and the triangles $t_{i} \cap S_{K}$.

Next, we define a graph $G_{r e d}$ in $S_{K}$ whose vertices are the barycenters of quadrangles or red triangles in $S_{K}$. The edges of $G_{r e d}$ are dual to red edges in $S_{K}$ in the following sense: each red edge $e$ in $S_{K}$ is incident on two cells, whose barycenters $\alpha$ and $\beta$ are vertices of $G_{r e d}$. Let $a_{e}$ be the arc in $S_{K}$ consisting of the two line segments connecting the barycenter of $e$ to $\alpha$ and $\beta$, respectively, see Fig. 7 .

Then $a_{e}$ connects $\alpha$ and $\beta$ and, clearly, the interiors of $a_{e}$ and $a_{f}$ are disjoint for any two different red edges $e$ and $f$. Hence $G_{r e d}$ is defined as a graph in $S_{K}$ by letting its edge set consist of the $\operatorname{arcs} a_{e}$, where $e$ is any red edge in $S_{K}$. Note that a vertex of $G_{r e d}$ is of order 2 or 3 depending on whether it is the barycenter of a quadrangle or a red triangle, respectively, see Fig. 8. Since the red boundary 


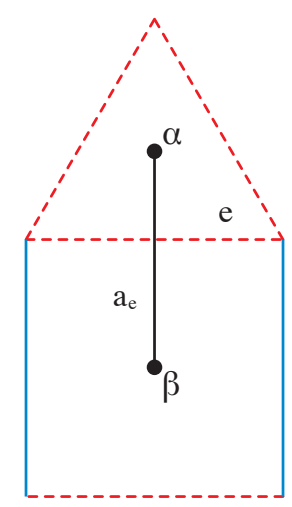

Figure 7: An edge in $G_{\text {red }}$ joining the centers of a red triangle and a quadrangle.

component $\partial K_{\text {red }}$ is connected it follows that the graph $G_{r e d}$ is connected.

Returning to the disc $\widetilde{B}_{r} \cap S_{K}$, we see that it contains the barycenters of the cells $t_{i j} \cap S_{K}$ and $t_{i} \cap S_{K}$ as well as the circuit $\gamma_{r}$ in $G_{r e d}$ made up of the edges connecting them. Clearly, $\gamma_{r}$ is a deformation of $\mu_{r}$ and likewise bounds an open disc $D_{r}$ in $S_{K}$, see Fig. 9.

By the construction of $\widetilde{B}_{r}$ in the proof of Lemma 2, the disc $D_{r}$ contains no part of $G_{r e d}$, and $D_{r} \cap D_{r^{\prime}}=\emptyset$ for $r \neq r^{\prime}$. Moreover, given a red edge $e$ in $S_{K}$ it is contained in a unique triangle in $K$ that contains a single edge $e^{\prime}$ in $\partial K_{\text {red }}$. Letting $r$ and $r^{\prime}$ be the endpoints of $e^{\prime}$ it follows that $a_{e}$ belongs to $\gamma_{r}$ and $\gamma_{r^{\prime}}$. This shows that the complement of $G_{r e d}$ in $S_{K}$ is a disjoint union of discs labelled by the vertices of $\partial K_{\text {red }}$. Hence, the Euler characteristic $\chi\left(S_{K}\right)$ of $S_{K}$ can be calculated by Euler's formula in terms of the graph $G_{r e d}$. If $F$ is the number of faces of $G_{r e d}, E$ the number of edges and $V$ the number of vertices we have

$$
\chi\left(S_{K}\right)=V-E+F .
$$

The number of faces of $G_{r e d}$ equals the number of vertices in $\partial K_{r e d}$ by the previous remark and the number of edges in $G_{r e d}$ equals the number of red edges in $S_{K}$ and fulfills

$$
2 E=3 \#\left\{\text { red triangles in } S_{K}\right\}+2 \#\left\{\text { quadrangles in } S_{K}\right\}
$$




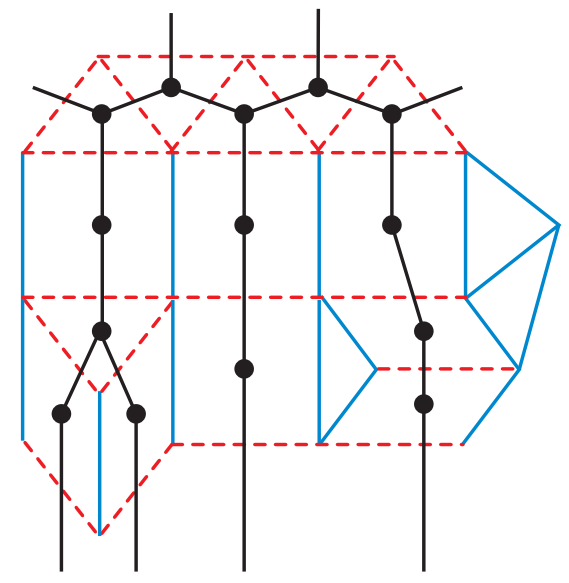

Figure 8: The graph $G_{r e d}$ corresponding to the midsection in Fig. 2.

Finally, the number of vertices in $G_{r e d}$ equals

$$
V=\#\left\{\text { red triangles in } S_{K}\right\}+\#\left\{\text { quadrangles in } S_{K}\right\}
$$

It follows that

$$
\begin{aligned}
\chi\left(S_{K}\right) & =\#\left\{\text { vertices in } \partial K_{\text {red }}\right\}-\frac{1}{2} \#\left\{\text { red triangles in } S_{K}\right\} \\
& =\chi\left(\partial K_{\text {red }}\right)
\end{aligned}
$$

since $\#\left\{\right.$ red triangles in $\left.S_{K}\right\}=\#\left\{\right.$ triangles in $\left.\partial K_{\text {red }}\right\}$ and

$$
2 \#\left\{\text { edges in } \partial K_{\text {red }}\right\}=3 \#\left\{\text { triangles in } \partial K_{\text {red }}\right\} \text {. }
$$

This completes the proof.

Remark. A proof of the lemma above can also be based on the existence of collar neighbourhoods of the boundary components (see, e.g., Corollary 2.26 in [15]), but we prefer to give the preceding more direct proof.

It can presumably be proven that a 3-dimensional generalized causal slice $K$ is homeomorphic to a cylinder. But we shall not need this fact and will not elaborate 


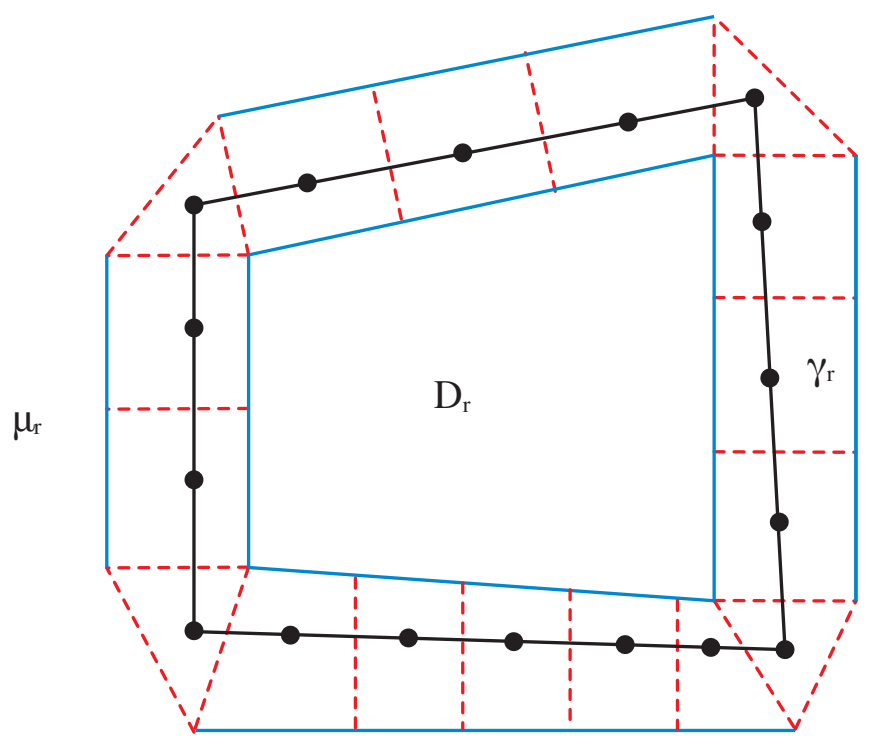

Figure 9: The curves $\mu_{r}$ and $\gamma_{r}$ and the disc $D_{r}$. In this case the red vertex $r$ has order 4.

further on it here, except for noting that in case the boundary components $\partial K_{\text {red }}$ and $\partial K_{\text {blue }}$ are homeomorphic to $S^{2}$ this fact follows easily from the validity of the Poincaré conjecture [14]. Indeed, it follows from Lemma 4 that the interior of $K$ is homeomorphic to $(0,1) \times S^{2}$. In particular, the interior of $K$ is simply connected. Being a manifold, $K$ has the structure of a cylinder close to its boundary by the collaring theorem. It follows that $K$ is also simply connected. By gluing two triangulated 3-balls onto the two boundary components of $K$ one therefore obtains a simply connected closed 3-manifold which is homeomorphic to the 3-sphere by the Poincaré conjecture. Removing the interior of the two 3-balls shows that $K$ is homeomorphic to a cylinder $[0,1] \times S^{2}$. Hence, in dimension 3 the notion of a generalized causal slice with boundary components homeomorphic to $S^{2}$ is equivalent to that of a causal slice.

Henceforth, we denote by $\mathcal{C}_{3, g}$ the set of generalized causal triangulations whose boundary components have genus $g$ and we let $N_{3, g}(V)$ denote the number of $K$ in 
$\mathcal{C}_{3, g}$ such that $\left|K^{3}\right|=V$. We then have the following generalization of Theorem 1 .

Theorem 3. There exists a constant $C_{3, g}>0$ such that

$$
N_{3, g}(V) \leq C_{3, g}^{V},
$$

for all positive integers $V$.

Proof. It is sufficient to verify the three steps in the proof of Theorem 1. Step (i) requires no change and the same applies to step (iii) since the topology of $S_{K}$ was not used to prove injectivity of $\pi$. In step (ii) the only modification required is the establishment of a bound on the number of triangulations of an oriented surface of fixed genus $g$ as a function of the number of triangles. This result may be found in [11] or, alternatively, it can easily be deduced from [17].

Remark. We note that the arguments in the proof of Theorem 2 apply to the case of boundaries with nonzero genus except that a suitable generalization of Lemma 3 is missing.

\section{The 4-dimensional case}

In this section we discuss the extension of Theorem 1 to the case of 4-dimensional causal triangulations. We let $M_{3}(V)$ denote the number of all abstract simplicial complexes homeomorphic to the 3 -sphere made up of $V$ tetrahedra and denote by $N_{4}(V)$ the number of 4-dimensional causal triangulations (as defined in Definition 1) made up of $V 4$-simplices. Our main result is the following.

Theorem 4. If there exists a constant $C>0$ such that $M_{3}(V) \leq C^{V}$ for all $V \geq 0$, then there exists a constant $C_{4}>0$ such that

$$
N_{4}(V) \leq C_{4}^{V},
$$

for all positive integers $V$.

Proof. We proceed in four steps. 
(i) By the same argument as in the proof of Theorem 1 it suffices to bound the number $N_{4}^{\prime}(V)$ of causal slices with $V$ 4-simplices.

(ii) Consider a causal slice $K$. By Lemma 1 , the midsection $S_{K}$ is a closed 3-manifold and the interior of $K$ is homeomorphic to $S_{K} \times(0,1)$ as well as to $S^{3} \times(0,1)$. In particular, $S_{K} \times(0,1)$ is simply connected and it follows that $S_{K}$ is simply connected and hence homeomorphic to $S^{3}$ by the Poincaré conjecture [14].

(iii) As explained in Section 2 the coloured cell complex $S_{K}$ has 3-cells of four types: red or blue tetrahedra and prisms whose end triangles are either both red or both blue. It is well known (see e.g. [15] Proposition 2.9) that any cell complex can be subdivided to a simplicial complex without introducing new vertices. Let $S_{K}^{\prime}$ be such a subdivision of $S_{K}$ and colour its new edges black. We note that each such edge $e$ connects two opposite vertices in a rectangular face $f_{e}$ of a prism of $S_{K}$ and that each prism $p$ of $S_{K}$ is subdivided in three tetrahedra all of which are incident on one of the three black edges in the boundary of $p$. In order to see that $S_{K}$ can be reconstructed from the coloured simplicial complex $S_{K}^{\prime}$ we further note that any black edge $e$ of $S_{K}^{\prime}$ is incident on exactly two triangles $\Delta_{e}, \Delta_{e}^{\prime}$ whose other edges are not black, and these triangles make up the face $f_{e}$. If $e$ is incident on three tetrahedra on one side of $f_{e}$ then those tetrahedra form a subdivision of a prism with $f_{e}$ as a face. These observations serve to show that the colouring of $S_{K}^{\prime}$ allows a reconstruction of the prisms of $S_{K}$ which together with the monocoloured tetrahedra define the cell complex $S_{K}$.

Noting that the number of 3-cells in $S_{K}$ equals $V$ and that the number of tetrahedra of $S_{K}^{\prime}$ is bounded by $3 V$, an exponential bound on the number of coloured cell complexes $S_{K}$ as a function of $V$ will follow from an exponential bound on the number of coloured simplicial complexes $S_{K}^{\prime}$ as a function of the number of tetrahedra. We obtain such a bound from (ii) and the assumed exponential bound on $M_{3}(V)$ since the number of possible colourings of the edges of a given simplicial manifold made up of $V$ tetrahedra is bounded by $9^{V}$.

(iv) If $S_{K}$ determines $K$ up to combinatorial isomorphism, that is if the map $\pi$ 
is injective on $\mathcal{C S}_{4}$, the claimed bound now follows from (i) and (iii). The proof of injectivity of $\pi$ is deferred to Lemma 5 below.

Lemma 5. Let $K \in \mathcal{C S}_{4}$. Then the coloured cell complex $S_{K}$ determines $K$ up to combinatorial isomorphism.

Proof. Given a causal slice $K \in \mathcal{C} \mathcal{S}_{4}$ with realization $K_{\phi}$ we construct from $S=S_{K}$ a 4-dimensional simplicial complex $K_{S}$ in complete analogy with the construction in the proof of Lemma 2 and whose details are left to the reader. The proof of the Lemma is complete once we show that $K_{S}$ is combinatorially isomorphic to $K$ and this in turn requires showing that if if $v_{1}, v_{2}$ are vertices of $S_{K}$ and $\bar{r}\left(v_{1}\right)=\bar{r}\left(v_{2}\right)$, using notation as in the proof of Lemma 2, then $v_{1}$ and $v_{2}$ are connected by a blue path and, similarly, if $\bar{b}\left(v_{1}\right)=\bar{b}\left(v_{2}\right)$ then $v_{1}$ and $v_{2}$ are connected by a red path. The proof of this fact follows by the same line of argument as in the the 3-dimensional case as we now describe.

Let $r$ be a vertex in $\partial K_{\text {red }}$ and consider the star $B_{r}$ of $r$ in $\partial K_{\text {red }}$, which is a 3-ball whose boundary $\partial B_{r}$ is a triangulated 2-sphere the triangles of which we denote by $\Delta_{1}, \ldots, \Delta_{n}$. The boundary tetrahedron $t_{i}=\left(r \Delta_{i}\right)$ is incident on a unique 4-simplex $s_{i}=\left(r b_{i} \Delta_{i}\right)$, and $r$ is not contained in any other 4-simplex of type $(4,1)$ except $s_{1}, \ldots, s_{n}$, since no mono-coloured interior edges exist in $K$.

Consider an edge $e$ in $\partial B_{r}$. The boundary triangle $(r e)$ is contained in exactly two tetrahedra $t_{e 1}=t_{i}$ and $t_{e 2}=t_{j}$ in $B_{r}$. If $b_{i} \neq b_{j}$ we repeat the construction applied in the 3-dimensional case to obtain a sequence $s_{e 1}, \ldots, s_{e m_{e}}$ of 4-simplices of type $(3,2)$ containing $(r e)$, such that $s_{e i}=\left(r b_{e(j-1)} b_{e j} e\right), j=1, \ldots, m_{e}$, and $b_{e 0}=b_{i}$ and $b_{e m_{e}}=b_{j}$. These are by construction different tetrahedra. Moreover, the triangle (re) is not contained in any other 4-simplex of type $(3,2)$ in $K$. In order to see this, observe that any such 4-simplex would intersect $s_{i} \cup s_{e 1} \cup \cdots \cup s_{e m_{e}} \cup s_{j}$ only in $(r e)$ by the assumption that any tetrahedron $\left(r b_{e k} e\right)$ is contained in exactly two 4 -simplices. It would follow that removing a small neighbourhood $N_{\epsilon}($ re $)$ from the star of $(r e)$ in $K$ one would obtain a disconnected set, contradicting the manifold 
property of $K$.

Repeating this construction for all edges $e$ in $\partial B_{r}$ we let $A_{0}$ denote the resulting simplicial complex consisting of the 4 -simplices $s_{i}, i=1, \ldots, n$, and $s_{e k}, k=$ $1, \ldots, m_{e}$, and their subsimplices. We let $C_{0}$ be the simplicial complex consisting of the edges $\left(b_{e(k-1} b_{e k}\right)$ and their vertices. We note that $C_{0}$ is a connected simplicial complex that equals the blue subcomplex of $A_{0}$. If we remove a sufficiently small neighbourhood of the 2 -skeleton of $A_{0}$ then a connected set remains. These properties are preserved under the subsequent constructions.

Pick now a vertex $v$ in $\partial B_{r}$ and let $f$ be an edge in $C_{0}$. If the tetrahedron $t=(r v f)$ is contained in exactly one 4 -simplex $s$ in $A_{0}$, then we define $A_{1}$ by adding to $A_{0}$ the other 4-simplex $s^{\prime}$ in $K$ that is incident on $t$. We let $C_{1}$ be obtained from $C_{0}$ by adding the triangle in $s^{\prime}$ opposite to $(r v)$. Clearly, this triangle contains $f$. If, on the other hand, either $t$ is not in $A_{0}$ or $t$ is incident on two 4-simplices in $A_{0}$ we set $A_{1}=A_{0}$ and $C_{1}=C_{0}$. Now repeat this step for some edge $f$ in $C_{1}$ to obtain $A_{2}$ and $C_{2}$ and continue until all tetrahedra in $A_{N}$ of the form $(r v f)$, where $f$ is an edge in $C_{N}$, are incident on two 4 -simplices in $A_{N}$. Then no further 4 -simplices of type $(2,3)$ in $K$ are incident on $(r v)$. In fact, such a simplex $(r v \Delta)$ would at most share three vertices with $C_{N}$ and hence at most three triangles with $A_{N}$, and we can use the same argument as above to conclude that the star of $(r v)$ in $K$, with a small neighbourhood of its 2-skeleton removed, would be disconnected, contradicting the manifold property of $K$.

Now repeat the construction for all vertices $v$ in $\partial B_{r}$ to obtain $A_{M}$ and $C_{M}$ such that $A_{M}$ contains all 4 -simplices of types $(4,1),(3,2)$ and $(2,3)$ in the star of $r$ in $K$ and such that $C_{M}$ is connected and equals the blue subcomplex of $A_{M}$. Finally, consider a triangle $\Delta$ in $C_{M}$. Then the tetrahedron $(r \Delta)$ is contained in some 4simplex of $A_{M}$ by construction. If the other 4-simplex of $K$ incident on $(r \Delta)$ is not in $A_{M}$ define $A_{M+1}$ by adding it to $A_{M}$ and define $C_{M+1}$ by adding its blue tetrahedron to $C_{M}$. Otherwise, $A_{M}$ and $C_{M}$ are left unchanged. Now continue repeating this construction untill $A_{L}$ and $C_{L}$ are obtained such that both 4-simplices in $K$ incident 
on $(r \Delta)$ are contained in $A_{L}$ for all triangles $\Delta$ in $C_{L}$, the blue subcomplex of $A_{L}$.

Then $A_{L}$ is the star of $r$ in $K$ : otherwise, there would be further 4-simplices of type $(1,4)$ in $K$ containing $r$ and these could at most share a union of triangles $(r e)$ or edges $(r v)$, where $e$ is an edge in $C_{L}$ and $v$ is a vertex in $C_{L}$, with $A_{L}$, and a contradiction is obtained in the same ways as previously.

Since $C_{N}$ is connected and its vertices coincide with the endpoints of edges originating from $r$ it follows that $\bar{r}\left(v_{1}\right)=\bar{r}\left(v_{2}\right)=r$ implies that $v_{1}$ and $v_{2}$ are connected by a blue path. Clearly, an analogous argument applies to any blue vertex $b$ instead of $r$ and the proof in complete.

Acknowledgements. This work was partly supported by the NordForsk researcher network "Random Geometry" (33000). We would like to thank Søren Galatius, Mauricio E. G. Lopez and Erik Kjær Pedersen for helpful discussions on piecewise linear topology. We are grateful to Ingi Freyr Atlason for help with the pictures.

\section{References}

[1] J. Ambjørn, B. Durhuus and T. Jonsson, Quantum geometry: a statistical field theory approach, Cambridge University Press, Cambridge (1997)

[2] J. Ambjørn, B. Durhuus and T. Jonsson, Three-dimensional simplicial quantum gravity and generalized matrix models, Mod. Phys. Lett. A 6 (1991) 1133-1146

[3] J. Ambjørn and R. Loll, Non-perturbative Lorentzian quantum gravity, causality and topolgy change, Nucl. Phys. B 536 (1998) 407-434

[4] J. Ambjørn, J. Jurkiewicz and R. Loll, Nonperturbative 3D Lorentzian quantum gravity, Phys. Rev. D 64 (2001) 044011

[5] J. Ambjørn, J. Jurkiewicz, R. Loll and G. Vernizzi, Lorentzian 3d gravity with wormholes via matrix models, JHEP 0109:022,2001

[6] J. Ambjørn, A. Görlich, J. Jurkiewicz and R. Loll, Nonperturbative quantum gravity, Physics Reports 519 (2012) 127210 
[7] J. Ambjørn and S. Varsted, Three-dimensional simplicial quantum gravity, Nucl. Phys. B 373 (1992) 557-577

[8] B. Benedetti and G. M. Ziegler, On locally constructible spheres and balls, Acta Math. 206 (2011) 205-243

[9] S. Catterall, J. Kogut and R. Renken, Entropy and the approach to the thermodynamic limit in three-dimensional quantum gravity, Phys. Lett. B 342 (1995) 53-57

[10] P. Collet, J.-P. Eckmann and M. Yonan, Trees of nuclei and bounds on the number of triangulations of the 3-ball, Commun. Math. Phys. 325 (2014) 259289

[11] B. Durhuus, Critical properties of some discrete random surface models. In proceedings of The 7'th Ettore Majorana International School of Mathematical Physics 1988, "Constructive quantum field theory II", eds. G. Velo and A.S. Wightman, Plenum Press, New York, 1990.

[12] B. Durhuus and T. Jonsson, Remarks on the entropy of 3-manifolds, Nucl. Phys. В 445 (1995) 182-192

[13] E. Hille and R. S. Phillips, Functional analysis and semi-groups, AMS, Providence (1957)

[14] G. Perelman, Finite extinction time for the solutions to the Ricci flow on certain three-manifolds, arXiv:math.DG/0307245

[15] C. P. Rourke and B. J. Sanderson, Introduction to picewise-linear topology, Springer, Berlin (1972)

[16] H. Seifert and W. Threlfall, A textbook of topology, Academic Press, New York (1980)

[17] W. T. Tutte, A census of planar maps, Can. J. Math. 15 (1963) 249-271 
[18] R. E. Williamson, Jr., Cobordism of Combinatorial Manifolds, Ann. Math. 83 (1966) 1-33 